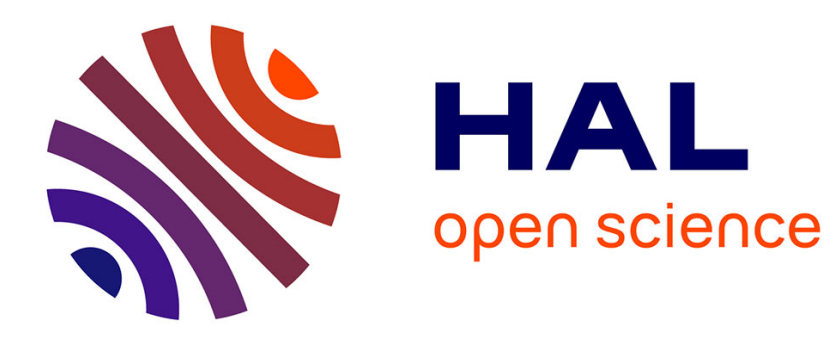

\title{
Présentation du $\mathbf{n}^{\circ} \mathrm{X}$ d'Ermeneutica letteraria
}

Donatella Bisconti, Elisabeth Kertesz-Vial

\section{- To cite this version:}

Donatella Bisconti, Elisabeth Kertesz-Vial. Présentation du n X d'Ermeneutica letteraria. Arzanà.

Cahiers de littérature médiévale italienne, 2017, 19, pp.79-82. 10.4000/arzana.1055 . hal-01988472

\section{HAL Id: hal-01988472 \\ https://hal.science/hal-01988472}

Submitted on 21 Jan 2019

HAL is a multi-disciplinary open access archive for the deposit and dissemination of scientific research documents, whether they are published or not. The documents may come from teaching and research institutions in France or abroad, or from public or private research centers.
L'archive ouverte pluridisciplinaire HAL, est destinée au dépôt et à la diffusion de documents scientifiques de niveau recherche, publiés ou non, émanant des établissements d'enseignement et de recherche français ou étrangers, des laboratoires publics ou privés. 


\section{Arzanà}

Cahiers de littérature médiévale italienne

19 | 2017

Varia

\section{Présentation du $\mathrm{n}^{\circ} \mathrm{X}$ d'Ermeneutica letteraria}

5 décembre 2014, Maison d'Italie - Paris; études réunies par Donatella Bisconti et Elisabeth Kertesz-Vial

Presentazione del $n^{\circ} X$ di Ermeneutica letteraria

Donatella Bisconti et Élisabeth Kertesz-Vial

revues.org

Édition électronique

URL : http://arzana.revues.org/1055

DOI : 10.4000/arzana.1055

ISSN : 2429-9499

\section{Éditeur}

Presses Sorbonne Nouvelle

\section{Édition imprimée}

Pagination : 79-82

ISSN : $1243-3616$

Ce document vous est offert par Centre national de la recherche scientifique (CNRS)

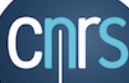

Référence électronique

Donatella Bisconti et Élisabeth Kertesz-Vial, « Présentation du n X d'Ermeneutica letteraria », Arzanà [En ligne], 19 | 2017, mis en ligne le 23 octobre 2017, consulté le 28 octobre 2017. URL : http:// arzana.revues.org/1055; DOI : 10.4000/arzana.1055

Presses Sorbonne Nouvelle 


\title{
Présentation du $\mathrm{n}^{\circ} \mathrm{X} \mathrm{d}^{\prime}$ Ermeneutica letteraria, 5 décembre 2014, Maison d'Italie - Paris; études réunies par Donatella Bisconti et Elisabeth Kertesz-Vial
}

\author{
Presentazione del $n^{\circ} X d i$ Ermeneutica letteraria
}

$\mathrm{L}$ a Table ronde du 5 décembre 2014 a réuni à la Maison d'Italie de Paris un nombre considérable de collègues italianistes français et italiens, d'étudiants et de personnes intéressées par les études italiennes de littérature et la philologie.

Mmes Élisabeth Kertesz-Vial et Donatella Bisconti, organisatrices de la journée, souhaitaient, par cette manifestation, prolonger le débat noué lors du colloque international Gianfranco Contini entre France et Italie : philologie et critique qui s'était tenu à Clermont-Ferrand du 30 mai au $1^{\text {er }}$ juin 2013 et dont les actes ont été recueillis dans le $\mathrm{n}^{\circ} \mathrm{X}$ de la revue Ermeneutica letteraria.

Paolo Leoncini, professeur émérite à l'université Ca' Foscari de Venise, qui avait lancé l'idée du colloque, avait vivement souhaité cette rencontre qui a permis de revenir sur les discussions que la manifestation de Clermont-Ferrand avait ouvertes, mais pas complètement épuisées. Bien au contraire, les participants au colloque avaient tous ressenti que leurs communications et le débat serré qui s'était ensuivi, loin de mettre un point final aux problématiques abordées, requerraient ce prolongement.

Le colloque avait fait notamment émerger chez Contini, d'une part, l'entrelacement entre le travail philologique et ecdotique consacré aux textes anciens et son activité critique tournée vers l'exploration de textes modernes et contemporains, et, d'autre part, le croisement entre les méthodes d'analyse littéraire et le travail sur les variantes et la mémoire intertextuelle. Ce qui frappe surtout, dans la longue carrière de Contini, c'est cet aller-retour incessant entre les méthodologies employées pour les textes anciens et celles appliquées aux textes et aux auteurs contemporains, qui se complètent réciproquement. En même temps, ce refus de confiner l'activité critique à une période ou à un groupe d'auteurs n'est pas l'indice d'un éclectisme ou, pire, d'un survol superficiel de thématiques diverses et variées, mais il marque plutôt la nécessité d'une extension progressive de la connaissance qui fait du texte, au sens étymologique du mot, le centre moteur d'une enquête en profondeur.

Cependant, l'attention de Contini au(x) texte(s), en tant qu'élément dynamique et évolutif dépassant la production d'un auteur et les limites entre les genres et les époques, ne saurait être séparée de son éthique de chercheur dont l'antifascisme se révèle, bien avant son entrée dans la Résistance aux côtés du mouvement "Giustizia e Libertà ", dans l'ensemble de ses choix éditoriaux (dont Italie magique constitue un spécimen emblématique), dans ses écrits théoriques, et dans son carteggio (sa 
correspondance). Elle se manifeste aussi par sa volonté obstinée de transmettre cet héritage - par la pédagogie - en rendant explicite le montage du texte et en permettant ainsi au lecteur l'accès au processus de sa mise en œuvre, tout en gardant une sorte de pudeur, d'autocensure, qui l'empêche de réduire le texte à une formula empruntée à Croce, ou d'exprimer sur celui-ci un jugement de valeur. Comme le rappellent tous nos contributeurs, le structuralisme et l'exigence expressive se conjuguent, donnant naissance à un grand élan critique. Dominique Budor, en particulier, prolonge la réflexion menée par Paolo Leoncini - et ce dès le premier numéro d'Ermeneutica letteraria ${ }^{1}$ - autour des « héritiers " de ce Contini novateur, qui allie les qualités d'empathie et d'intuitivité aux qualités scientifiques. Comment ne pas citer Paul Ricœur, dont la proximité de la pensée sur ce point rejoint le travail de Contini : "C'est [...] la tâche de l'herméneutique de reconstruire l'ensemble des opérations pour lesquelles une œuvre s'enlève sur le fond opaque du vivre, de l'agir et du souffrir pour être donnée par un auteur à un lecteur qui la reçoit et ainsi change son $\operatorname{agir}^{2}$ ».

En effet, la pédagogie continienne - axée à la fois sur les aspects matériels objectifs (données philologiques, tradition du texte, variantes d'auteur...) et des aspects moins palpables, de type émotionnel, mais également vérifiables par le biais de l'analyse linguistique - réactive sans cesse un cercle herméneutique et justifie scientifiquement la corrélation méthodologique entre le passé et le présent.

C'est à partir de ces constats que les participants à la Table ronde du 5 décembre 2014 ont voulu, chacun selon l'angle d'attaque propre à ses spécialisations critiques, non seulement rendre compte du $\mathrm{n}^{\circ} \mathrm{X}$ d'Ermeneutica letteraria et ses différentes contributions, mais s'approprier la leçon de Contini : leurs apports à la discussion constituent à la fois une synthèse des positions qui ont émergé lors du colloque de Clermont-Ferrand et une ouverture vers de nouvelles perspectives restées implicites et potentielles dans les travaux du domese ${ }^{3}$, mais toujours susceptibles de développements, comme le montrent d'ailleurs plusieurs travaux accueillis dans le ${ }^{\circ} \mathrm{X} \mathrm{d}^{\prime}$ Ermeneutica letteraria ainsi que les contributions de Viviana Agostini-Ouafi et Dominique Budor présentées ci-après.

C'est dans cette perspective que nous avons choisi d'intituler cet ensemble de travaux Scavi continiani, une paraphrase, comme tout lecteur de Contini l'aura compris, des Scavi alessiani ${ }^{4}$, en quelque sorte un prolongement aujourd'hui de la méthode du philologue et du critique.

L'intervention de Raffaella Zanni place au cour de l'activité critique de Contini l'« assillo di verità ", qui se traduit dans son éros pédagogique alliant philologie et critique militante et inversant la logique de l'apprentissage du présent par le passé. Le présent devient en effet propédeutique à la compréhension des manifestations littéraires anciennes, puisqu'il ne requiert pas la reconstitution préalable d'une culture "dalla a alla zeta" ». De ce fait, comme le remarque, sur la base des travaux d'Enza Biagini, Paolo Leoncini, dans les pages qui suivent, synchronie et diachronie ne sont pas conflictuelles, mais s'allient dans le microcosme des " esercizi " continiens, tandis que la philologie prime sur la philosophie (ou, si l'on veut, l'idéologie, voire les idéologismes), en d'autres termes, l'appréhension théorique est parallèle à l'intuition du phénomène linguistique qui l'alimente, et lui fournit sa justification éthique.

Honnêteté et pragmatisme, voilà la formule qui résume G. Contini. On la doit à Emilio Cecchi, comme le rappelle Paolo Leoncini qui insiste sur le côté démocratique du philologue. C'est sans

1. Paolo LEONCINI, «Interpretazione e verità : variabili ermeneutiche di Gianfranco Contini », Ermeneutica letteraria, $\mathrm{n}^{\circ} \mathrm{I}, 2005$, p. 53-79.

2. Paul RiCOEUR, Temps et récit 1, Paris, Éd. du Seuil, coll. «Points Essais », 1983, p. 106-107.

3. Domese : ainsi nomme-t-on en italien un habitant de Domodossola.

4. Gianfranco CONTINI, "Scavi alessiani ", in Linguistica e Filologia, Omaggio a Benvenuto Terracini, a cura di Cesare Segre, Milano, Il Saggiatore, 1968, p. 59-95, ensuite in ID., Breviario di ecdotica, Torino, Einaudi, 1990, p. 99-134.

5. V. Gianfranco CONTINI, Un anno di letteratura, Firenze, Le Monnier, 1942, p. 152, désormais in ID., Esercizi di lettura, Torino, Einaudi, 1982, p. 387-388. 
aucun doute la matrice rosminienne du domese qui explique cette attention aux formes dialectales les plus populaires et à l'appréciation des premiers poèmes d'un Pier Paolo Pasolini, où conscience politique et exercice littéraire s'entrelacent.

En ce sens, la perspective de Contini ne pouvait qu'être supranationale et européenne : la coexistence de synchronie et diachronie et l'élargissement des horizons du philologue au-delà de la frontière italienne correspondent en effet à une proposition politique qui englobe et dépasse en même temps l'opposition à la politique culturelle du fascisme pour atteindre une dimension gnoséologique propre à une critique littéraire antidogmatique refusant, par là même, tout enfermement dans des savoirs spécialisés et cloisonnés, comme Raffaella Zanni le souligne vers la fin de sa contribution. Viviana Agostini-Ouafi va dans le même sens en insistant sur les rapports entre Pézard et les dantologues français et en présentant les travaux contrastifs sur la question de l'interprétation et de la traduction. Les contributions ici réunies prolongent également les débats du colloque de Clermont-Ferrand en approfondissant la question des liens tissés, tant dans l'espace que dans le temps, des deux côtés des Alpes, autour de l'interprétation.

Le plaisir du texte ou, mieux, des textes, mais également l'intérêt, voire l'amour - D. Budor cite, pour illustrer la vocation à la fois critique et militante de Contini, l'affirmation selon laquelle "Ogni problema pedagogico è d'amore, da Platone in giù ${ }^{6}$ ". Cette ouverture vers d'autres disciplines - les arts, la musique, la philosophie, les sciences naturelles - permet à Contini d'appliquer la notion de système, qu'il emprunte à Ferdinand de Saussure, à la philologie romane tout d'abord : la tradition manuscrite est un ensemble où chaque témoin interagit dans un parcours qui se déroule en aval, mais également sur l'axe synchronique, en d'autres termes sur d'autres témoins contemporains. La rigidité de la philologie lachmanienne est ainsi réactualisée grâce aux apports de Giorgio Pasquali en ce qui concerne les textes classiques, de Joseph Bédier et de Santorre Debenedetti pour les textes médiévaux : de Debenedetti il apprend la pratique du métier, de tout métier, comme idéal, comme volonté de prendre conscience de soi, ce qui, soit dit en passant, rapproche l'éthique de Contini, cette éthique à la fois professionnelle et existentielle, de l'attitude d'un Primo Levi. Ce dernier, né seulement quelques années après Contini, n'abandonna jamais dans son œuvre critique et littéraire l'exercice presque acharné de la raison, en dépit - ou peut-être à cause - de l'expérience dévastatrice de la déportation.

L'éthique du philologue roman consiste tout d'abord à reconnaître que le texte n'est pas un produit fini et établi à jamais, mais qu'il est un «work in progress " par définition. Étudier l'histoire d'une tradition manuscrite revient donc à s'intéresser au diasystème (linguistique, mais aussi culturel, graphique, éditorial, poétique) qui fait du texte à la fois un unicum et un tissu de facteurs multiples permettant l'intervention du critique, là où les variantes textuelles éclairent son devenir.

À propos de la Variantistica d'aujourd'hui et de demain, D. Budor conclut sur des vœux que nous voulons partager avec les lecteurs de la revue Arzanà :

Dans la reconstitution de la genèse', c'est certes le rapport au temps et à l'espace de la création qui constitue le nœud problématique de l'analyse. Or sur ce point, tant les 'Continiens' que les généticiens pourront sans doute imaginer des synergies nouvelles entre les différentes approches, à l'heure où les procédures d'édition numérique et les nouvelles technologies scientifiques du déchiffrement des strates autographes offrent l'espoir de lire les manuscrits, et de les éditer, dans toute la profondeur de leurs couches spatiales et temporelles. ${ }^{8}$

6. Gianfranco CONTINI, Esercizî di letteratura sopra autori contemporanei con un'appendice su testi non contemporanei. Nuova edizione aumentata di "Un anno di letteratura", Torino, Einaudi, 1974, p. 389.

7. Sur ce point, rappelons aussi les commentaires de Paolo Leoncini sur « les valeurs à naître " continiennes.

8. V. ci-après, p. 112. 
L'« approssimazione al valore ", comme Contini définit la tentative de reconstituer le réseau sous-tendant le texte, tout en s'inspirant de la notion de valeur - alternative à celle de signe - issue de la linguistique saussurienne, marque également l'écart par rapport à cette tradition pour laquelle la langue en tant que «système de valeurs » ou, en d'autres termes, de relations arbitraires entre la forme et la substance du signifié, obéit tout de même à des normes que le locuteur accepte sans les remettre en question. En revanche, pour le domese, se rapprocher de la valeur implique d'une part une tension incessante du travail critique vers l'interprétation du texte et, d'autre part, la prise de conscience que l'exigence herméneutique constitue le terrain d'entente entre le critique et le lecteur. Cela implique, entre autres, le rejet de la formula de Croce en tant que jugement sur le texte comme produit définitivement achevé, dont la signification serait établie à jamais.

C'est sur ce plan que Viviana Agostini-Ouafi affirme justement qu'il existe pour Contini une connexion entre « lo sviluppo espressivo dinamico, [...] iscritto nella storia » et la "struttura [...] insieme di corrispondenze sistematiche, in apparenza statiche e sincroniche che sono strettamente connesse al commento esegetico ». La relation dialectique entre les deux aspects se joue ici au sein de la critique dantesque, mais l'opération est extensible à d'autres moments et à d'autres auteurs de l'histoire littéraire, en tant que " critica verbale » qui passe par une lecture réitérée du texte, non seulement selon les modalités prônées par la critique stylistique, mais surtout pour en faire émerger une "traduzione inedita " : la transposition d'une valeur sémantique à une autre, justifiable sur l'axe diachronique - comme le montre l'analyse célèbre du sonnet de Dante Tanto gentile - mais également sur l'axe synchronique, lorsque la lecture se concentre sur un texte contemporain, dont le développement reste ouvert et où les échos demandent à être décodés.

En explorant la relation Contini-Pézard, Viviana Agostini-Ouafi souligne l'appréciation du philologue français pour l'alliance chez Contini entre la rigueur de la critique textuelle et la critique interprétative qui en découle. L'appareil critique, dont Contini enrichit ses éditions de textes anciens, fait état d'une érudition parfaitement assimilée, visant à guider la compréhension du lecteur, un lecteur actif qui doit contribuer à la réflexion sur le texte.

On peut souligner qu'il s'agit d'une philologie s'adressant à tous les lecteurs, même aux non spécialistes, une philologie qui n'a pas pour seul objectif de passer le relais à d'autres maîtres qui resteraient isolés dans leur tour d'ivoire, mais de forger des élèves en mesure d'en élargir et d'en développer l'héritage, de dépasser, pourrait-on ajouter, la limite propre à la culture italienne, celle que Graziadio Isaia Ascoli dénonçait dans le Proemio dell'Archivio glottologico italiano : « la scarsa densità della cultura e l'eccessiva preoccupazione della forma ", conséquence de la présence de maîtres certes excellents, mais qui brillent comme des monades isolées et ne sont pas suivis par la multitude d'élèves à laquelle on pourrait s'attendre? .

Pourtant, le langage critique de Contini, malgré son intention pédagogique, surprend le lecteur non averti par sa complexité qui s'apparente parfois à de l'obscurité, comme si le texte critique luimême demandait une intervention exégétique de la part du lecteur et, tout comme le texte littéraire, sollicitait une activité herméneutique semblable à un dialogue entre l'auteur et ses lecteurs potentiels, tel qu'il s'est réellement produit dans la réalité, si l'on réfléchit aux échanges constants entre Contini et les auteurs contemporains dont il s'est occupé dès sa jeunesse.

Dans cette livraison d'Arzanà, on voudrait souligner que se précise encore un peu mieux la figure dessinée lors du colloque clermontois, ce nouveau Contini qui avait émergé dès les années soixante, et que la revue Ermeneutica letteraria avait fait reconnaitre et apprécier.

9. Graziadio Isaia ASCOLI, "Proemio all'Archivio Glottologico Italiano », in ID., Scritti sulla questione della lingua, a cura di Corrado Grassi, con un saggio di Guido Lucchini, Torino, Einaudi, 2008. 